\title{
Theoretical Understanding of the Linear Relationship between Convective Updrafts and Cloud-Base Height for Shallow Cumulus Clouds. Part II: Continental Conditions
}

\author{
YOUTONG ZHENG \\ Earth System Science Interdisciplinary Center, University of Maryland, College Park, College Park, Maryland \\ MIRJANA SAKRADZIJA \\ Max Planck Institute for Meteorology, Hamburg, Germany \\ SEOUNG-SOO LEE \\ Research Foundation, San Jose State University, San Jose, California \\ ZHANQING LI \\ Earth System Science Interdisciplinary Center, University of Maryland, College Park, College Park, Maryland
}

(Manuscript received 28 October 2019, in final form 3 February 2020)

\begin{abstract}
S
This is the Part II of a two-part study that seeks a theoretical understanding of an empirical relationship for shallow cumulus clouds: subcloud updraft velocity covaries linearly with the cloud-base height. This work focuses on continental cumulus clouds that are more strongly forced by surface fluxes and more deviated from equilibrium than those over oceans (Part I). We use a simple analytical model for shallow cumulus that is well tested against a high-resolution $(25 \mathrm{~m}$ in the horizontal) large-eddy simulation model. Consistent with a conventional idea, we find that surface Bowen ratio is the key variable that regulates the covariability of both parameters: under the same solar insolation, a drier surface allows for stronger buoyancy flux, triggering stronger convection that deepens the subcloud layer. We find that the slope of the Bowen-ratio-regulated relationship between the two parameters (defined as $\lambda$ ) is dependent on both the local time and the stability of the lower free atmosphere. The value of $\lambda$ decreases with time exponentially from sunrise to early afternoon and linearly from early afternoon to sunset. The value of $\lambda$ is larger in a more stable atmosphere. In addition, continental $\lambda$ in the early afternoon more than doubles the oceanic $\lambda$. Validation of the theoretical results against ground observations over the Southern Great Plains shows a reasonable agreement. Physical mechanisms underlying the findings are explained from the perspective of different time scales at which updrafts and cloud-base height respond to a surface flux forcing.
\end{abstract}

\section{Introduction}

Shallow cumulus clouds exert strong net radiative forcing to the climate system (Hartmann et al. 1992). Cumulus clouds are profoundly influenced by subcloud updrafts in many ways (Donner et al. 2016; Emanuel 1994; Stull 2012), but measurements of updrafts are still scant. Zheng and Rosenfeld (2015) have suggested a remote sensing method to infer the subcloud updraft speed from cloud-base height $z_{b}$ that is easier to obtain. This concept is supported by an observed correlation between the two variables for shallow cumulus over the

Corresponding author: Youtong Zheng, zhengyoutong@gmail.com
Southern Great Plains (SGP), Amazon regions, and northeast Pacific. Such a relationship is not only useful for updraft remote sensing, but also for understanding moist convection processes as the subcloud updrafts and $z_{b}$ set the stage for the development of in-cloud convection (e.g., Emanuel 1991; Williams and Stanfill 2002).

This paper is Part II of a two-part study that aims to establish a theoretical basis for the empirically observed linear covariation between subcloud updraft speed and $z_{b}$. In Zheng (2019, hereafter Part I), a theoretical framework has been established for oceanic shallow cumulus. The theory suggests that this relationship arises from the conservation law of energetics: radiative fluxes divergence of a subcloud mixed layer (ML) has 
to balance surface heat fluxes. Given a certain ML radiative cooling rate per unit mass $(Q)$, a deeper $\mathrm{ML}$ (higher $z_{b}$ ) undergoes greater divergence in radiative fluxes and requires stronger surface fluxes to balance it, leading to stronger updrafts. The increasing rate of updrafts with the $z_{b}$ is modulated by $Q$. The rate $Q$ is proved to be resilient to large variations of external large-scale forcing, causing the relationship to appear linear.

Unlike marine boundary layers that can be considered quasi-stationary, boundary layers over continents rarely reach equilibria because of the fast varying surface heat fluxes forced by diurnal solar forcing. Under such nonstationary surface forcing, a cumulus-capped boundary layer is essentially a transient system that is not constrained by the boundary layer radiative-convective equilibrium (this equilibrium should be distinguished from the classical radiative-convective equilibrium for the entire troposphere). Why do observations still show tightly linear updrafts $-z_{b}$ relationship over land (Zheng and Rosenfeld 2015)? A conventional idea is the surface Bowen ratio (BR) regulating both parameters (Williams and Stanfill 2002). Given the same degree of solar insolation, higher BR corresponds to stronger surface sensible heat flux that drives stronger convection. The stronger convective overturning, in turn, enhances the entrainment rate near the ML top, deepening the ML. This BR-driven covariation of updrafts and ML depth has been known in large-eddy simulation (LES) studies (Golaz et al. 2001). What has been less known is the slope of the covariation. If we use the Deardorff velocity scale $w^{*}$ (Deardorff 1970) and subcloud ML depth $h$ to approximate the updraft strength and cloud-base height, respectively, the quantity of interest is the $\lambda=d w^{*} d h$, where the $d w^{*}$ and $d h$ correspond to changes between land surfaces with different BR. Note that the changes here refer to those caused by changes in surface BR, not temporal changes at a subdiurnal time scale. This differentiation can be illustrated by the schematic diagram in Fig. 1, which shows hypothetical daytime evolutions of $w^{*}$ and $h$ over two land surfaces with different BR. The BR is assumed to be constant within a day (Gentine et al. 2011, 2007). For a given BR, $w^{*}$ and $h$ still change with time because of the diurnally changing surface solar forcing. These temporal changes, however, are not relevant to the $d w^{*}$ and $d h$ in the $\lambda$ definition, which dictate changes between different BR. Despite the diurnally invariant BR, the $\lambda$ is possible to have a diurnal cycle. As shown in the Fig. 1, the $\lambda\left(t_{1}\right)=\Delta w *\left(t_{1}\right) / \Delta h\left(t_{1}\right)$ can be different from the $\lambda\left(t_{2}\right)=\Delta w^{*}\left(t_{2}\right) / \Delta h\left(t_{2}\right)$, and the extent of the difference depends on physical processes governing the diurnal behaviors of each parameter.
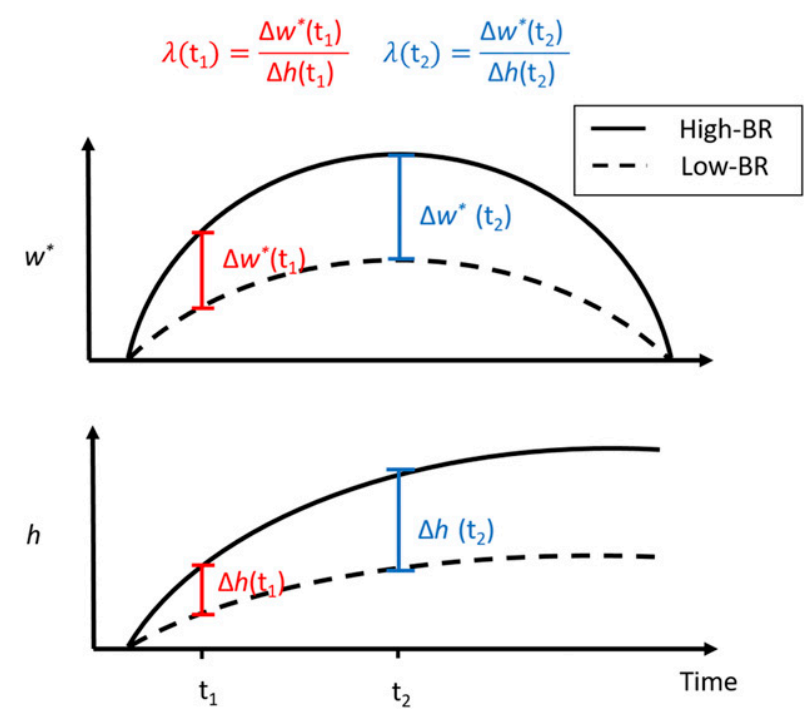

FIG. 1. Schematic diagram illustrating the definition of $\lambda$ and its diurnal dependence.

This study examines $\lambda$ for continental shallow cumulus. In particular, we are interested in three scientific questions:

1) How does $\lambda$ vary in different stages of a daytime diurnal cycle?

2) How does $\lambda$ vary in different thermodynamic conditions of the atmosphere?

3) Is $\lambda$ over land greater or smaller than that over ocean?

For the third question, the experimental data (Zheng and Rosenfeld 2015) suggest that the continental $\lambda$ is $\sim 20 \%$ greater than that over the ocean. However, due to considerable scatters of the data samples and due to a lack of theoretical justification, Zheng and Rosenfeld (2015) did not call attention into such a land-ocean difference. Progress in theoretical understanding of this question has been achieved in Part I of this study, paving the ground for the current study.

We will use the same analytical ML model for shallow cumulus (Neggers et al. 2006) as in Part I. Although this model is originally developed for the equilibrium marine boundary layer, it proves to be applicable to continental shallow cumulus with surprisingly good performance (van Stratum et al. 2014). In Part I, we use $w^{*}$ to approximate the strength of updraft. This approximation, however, is less valid for a boundary layer with nonstationary surface fluxes (van Driel and Jonker 2011), in particular in the decaying stage of continental cumulus near sunset. We will address this problem by revising $w^{*}$ in a physical way and validating the revised $w^{*}$ against LES. 


\section{A prototype problem: The encroachment growth}

Before investigating the problems with full complexity, we start with a simpler problem. We consider a thermally stratified fluid driven by a constant buoyancy flux from below. By neglecting the penetrative entrainment, the evolution of the buoyancy-driven $h$ follows the well-known "encroachment" growth (Stull 2012):

$$
h(t)=\left(\frac{2 F_{B}}{\gamma_{\theta_{v}}} t\right)^{1 / 2},
$$

where $F_{B}$ is the surface buoyancy flux and $\gamma_{\theta_{v}}$ is the lapse rate of virtual potential temperature $\theta_{v}$ of the background fluid. The vertical velocity of the system can be characterized by $w^{*}$ (Deardorff 1970):

$$
w^{*}=\left(\frac{g h}{\theta_{v}} F_{B}\right)^{1 / 3},
$$

where $g$ is the acceleration of gravity. Combining Eq. (1) with Eq. (2) yields

$$
w^{*}(t)=\left(\frac{g \gamma_{\theta_{v}}}{2 \theta_{v} t}\right)^{1 / 3} h=\left(\frac{N^{2}}{2 t}\right)^{1 / 3} h,
$$

where $N=\left[\left(g / \theta_{v}\right) \gamma_{\theta_{v}}\right]^{1 / 2}$ is the Brunt-Väisälä frequency. For a given $N, F_{B}$ is the sole determinant of $h$ and $w^{*}$, in which case $\lambda=\left(N^{2} / 2 t\right)^{1 / 3}$. Figure 2 visualizes $\lambda$ as a function of $t$ and $N$. The value of $\lambda$ decreases exponentially with time. In addition, $\lambda$ increases with increasing $N$, which means that the $w^{*}-h$ slope is steeper in a more stable atmosphere. The results suggest answers to the first two questions posed in the introduction.

The above analysis also suggests that the $w^{*}-h$ relationship manifests linearity for a given $N$. That is to say, linearity emerges when the variation in $h$ is solely determined by changes in $F_{B}$. In the real atmosphere, however, both $F_{B}$ and $N$ vary, jointly controlling $h$ (Santanello et al. 2005, 2007). It is, therefore, worthwhile to explore how $w^{*}$ varies with $h$ in a more realistic system with both parameters varying. To that end, we take the total derivative of $w^{*}$ with respective to $h$ in Eq. (3):

$$
\frac{d w^{*}}{d h}=\frac{\partial w^{*}}{\partial h}+\frac{\partial w^{*}}{\partial N} \frac{\partial N}{\partial h} .
$$

If $N$ is fixed, the second term in Eq. (4) is equal zero and $d w^{*} / d h=\partial w^{* / \partial h}=\left(N^{2} / 2 t\right)^{1 / 3}$, which describes the same system as that in Fig. 2. If $N$ is allowed to vary, Eq. (4), with several steps of derivations (see detail in appendix A), becomes

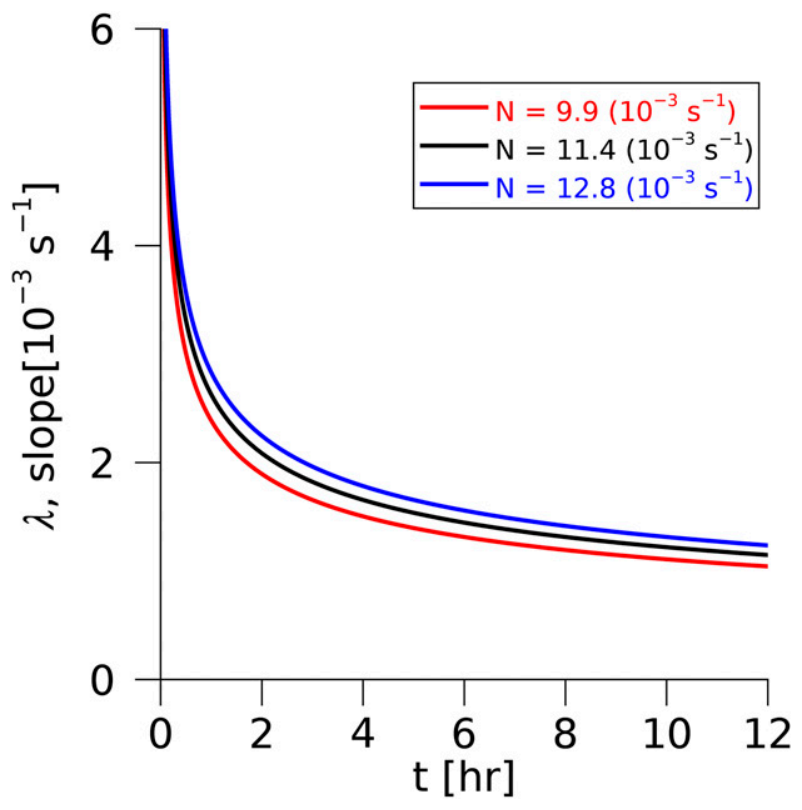

FIG. 2. Temporal variation of slopes of $w^{*}$ vs $h$ for different Brunt-Väisälä frequencies $N$.

$$
\frac{d w^{*}}{d h}=\left(\frac{N^{2}}{2 t}\right)^{1 / 3}-\frac{2}{3}\left(\frac{N^{2}}{2 t}\right)^{1 / 3}=\frac{1}{3}\left(\frac{N^{2}}{2 t}\right)^{1 / 3} .
$$

Equation (5) suggests a nonlinear $w^{*}-h$ relationship because $N$ is an independent variable, with which $h$ changes. We visualize the mathematical analysis by plotting a $w^{*}-h$ space at $t=8 \mathrm{~h}$ (Fig. 3a). We perturb $N$ by $\Delta N$ while holding $F_{B}$ constant (blue arrow), and do the same thing for $F_{B}$ (orange arrow). The value of $w^{*}$ increases with $h$ in a linear way if $F_{B}$ is varied, and in a cubic-root way if $N$ is varied.

Therefore, it is important to know the relative importance of surface fluxes and atmospheric stability contributing to the $h$ variation. Toward that end, we use the ground-based observations over the SGP from Zheng and Rosenfeld (2015). There are a total of 209 shallow cumulus cases manually selected from state-of-art ground-based remote sensors between 2010 and2014. Each case corresponds to a 3-h segment of measurements. Local times of most cases fall between noon and early afternoon when shallow clouds were developing actively. We calculate $\gamma_{\theta_{v}}$ from the early morning sounding at 1130 UTC. An atmospheric segment between the early morning planetary boundary height [determined from Liu and Liang's (2010) methodology] and $3.5 \mathrm{~km}$ was selected for calculating $\gamma_{\theta_{v}}$. Data on surface fluxes are from the quality-controlled eddy correlation flux measurements over the SGP site. We use the surface sensible heat flux $F_{\mathrm{SH}}$ (a good proxy for $F_{B}$ over continent) averaged from the sunrise to the sampling time of each case 

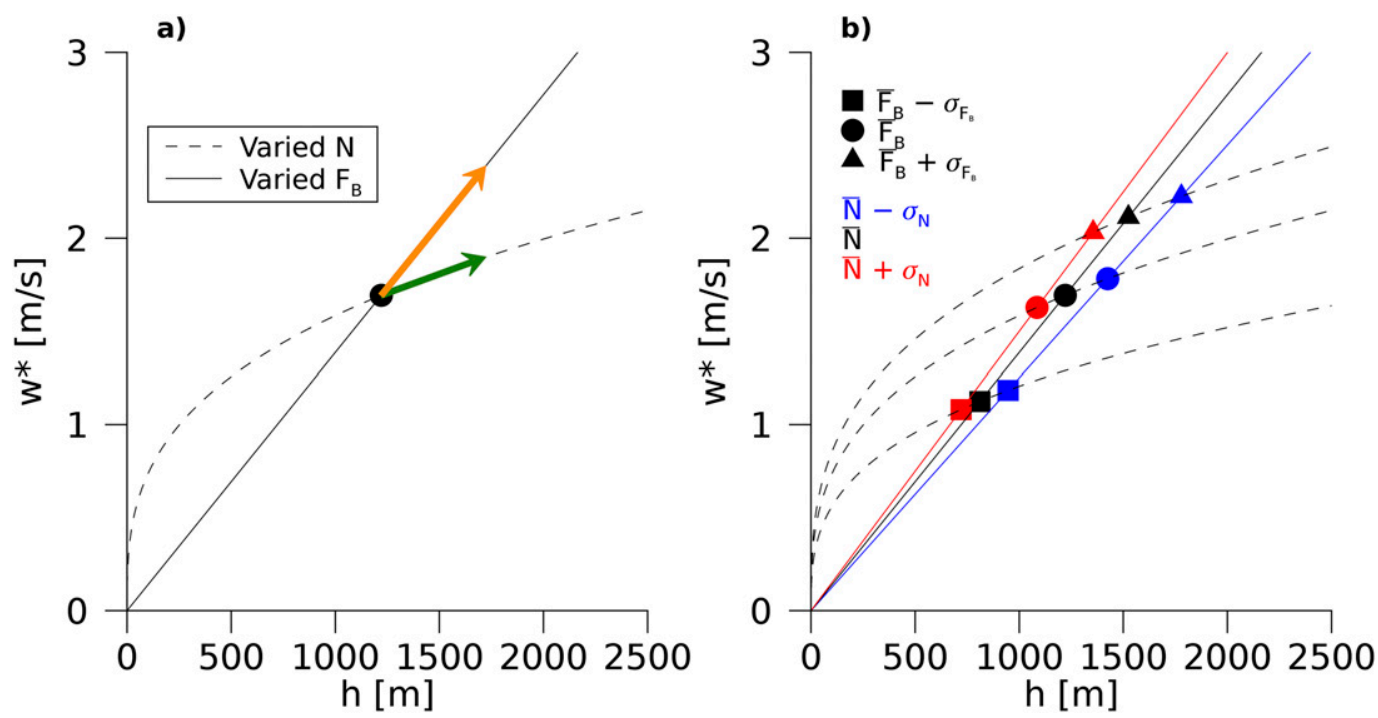

FIG. 3. Relationships between $w^{*}$ and $h$ at $t=8 \mathrm{~h}$, calculated by the "encroachment" framework. (a) Illustration of different ways with which $w^{*}$ varies with $h$ when only $N$ or $F_{B}$ is varied. (b) Distribution of idealized cases constructed from statistics of SGP cases. The overline symbol and $\sigma$ represent the composite mean and standard deviation, respectively. The solid and dashed lines represent the relationships for varied $N$ and varied $F_{B}$, respectively.

to represent the impacts of surface forcing. The composite mean for $F_{\mathrm{SH}}$ is $147 \pm 83 \mathrm{~W} \mathrm{~m}^{-2}$ and for $\gamma_{\theta_{v}}$ is $4.7 \pm 1.2 \mathrm{~K} \mathrm{~km}^{-1}$. To obtain an intuitive sense of how the observed spreads in $F_{\mathrm{SH}}$ and $\gamma_{\theta_{v}}$ contribute to the $w^{*}-h$ covariation, we input their composite statistics (mean plus and minus the standard deviation) to the simple encroachment model at $t=8 \mathrm{~h}$ (Fig. 3b). The first thing to notice is that $F_{B}$ contributes more to the $h$ variance than $N$. This result is physically reasonable because cloud regimes are typically associated with a certain range of atmospheric stability (Tselioudis et al. 2013; Wood and Bretherton 2006). It is very rare for broken cumulus clouds to form under very stable atmospheric conditions in which the strong stratification favors stratiform clouds (Wood and Bretherton 2006) or, if strong enough, no clouds. Likewise, very unstable atmosphere is favorable for the vertical development of convective clouds so that shallow convection evolves into deep ones. This restriction markedly limits the variability of atmospheric stability for shallow cumulus clouds $\left(\gamma_{\theta_{v}}\right.$ varies by only $\pm 27 \%$ whereas $F_{\mathrm{SH}}$ varies by $\pm 56 \%$ ). Simple correlative analysis of the 209 cases shows that $\gamma_{\theta_{v}}$ explains only $\sim 18 \%$ of the $h$ variance whereas $F_{\mathrm{SH}}$ explains $\sim 47 \%$.

The greater contribution of $F_{B}$ to the $h$ variance than $N$ makes the overall $w^{*}-h$ relationship appear closer to linearity with a certain degree of scattering induced by the variance in $N$ (Fig. 3b). In other words, the linear $w^{*}-h$ covariation driven by changes in BR (solid line in Fig. 3a) is a good first-order fit to the overall $w^{*}-h$ relationship. As such, in the remainder of the manuscript, $\lambda$ is evaluated for a system with varied BR only. The $N$ is considered an environmental parameter that is fixed for the system, but may vary among systems. Strictly speaking, we should use a new symbol (e.g., $\lambda_{\mathrm{BR}}$ ) to denote the assumption that only BR is varied for deriving the $w^{*}-h$ slope. Here we keep using $\lambda$ for simplicity.

\section{Hypotheses}

The results from the simple "encroachment" theory (Fig. 2) can help us to hypothesize answers to the first two questions posed in the introduction:

1) The values of $\lambda$ decreases over time.

2) The values of $\lambda$ is greater in a more stable atmosphere.

Formulations of these hypotheses are based upon the following assumptions:

- Early morning residual layer, which may suddenly deepen the boundary layer, is not considered.

- The time scale of change of surface Bowen ratio is markedly longer than the subdiurnal time scale (so-called daytime self-preservation of evaporative fraction).

- Static stability of the large-scale background atmospheric flow is not correlated with the surface Bowen ratio.

- Convective velocity scale can represent the strength of subcloud updrafts over land.

- Impacts of penetrative entrainment on $\lambda$ are not accounted for. 
- Impacts of cloud mass fluxes on $\lambda$ are not accounted for.

To make the theory analytically solvable, the first two assumptions have to be made, which are not uncommon in theoretical studies of continental boundary layers (e.g., Betts 2000; Gentine et al. 2013; Sakradzija and Hohenegger 2017). Strictly speaking, the assumption of daytime constant evaporative fraction is only valid during midday (roughly between 1000 and 1500 local time) (Gentine et al. 2007). Here, we assume no diurnal variation for analytical simplicity. The third assumption should not hold in general. For example, occurrence of a high pressure system over a region (more stable atmosphere) reduces cloudiness and precipitation. This depletes soil moisture, thereby increasing the Bowen ratio (Miralles et al. 2014). For the shallow cumulus regime, however, we do not expect such a land-atmosphere coupling to be effective because a lack of precipitation is a typical characteristic for shallow cumuli. This argument is confirmed by our analysis of 209 SGP shallow cumulus cases, which shows no statistically significant correlation between $N$ and $F_{\text {SH. }}$.

The last three assumptions will be dealt with in the following section, where we use a more sophisticated ML model that is tested against a LES model.

\section{Neggers et al.'s (2006) analytical model and its validation}

Following Part I of this study, the theoretical exploration in this study is primarily based on an analytical mixed-layer model (MLM) for shallow cumulus developed by Neggers et al. (2006). Readers can refer to appendix B for the description of this model. An important merit of a simple analytic model is that it captures the essence of a physical problem while remaining analytically tractable (Jeevanjee et al. 2017). Also it is computationally cheap. This MLM is originally developed for equilibrium shallow cumulus, but it performs well over continent in terms of its ability to simulate diurnal variations of the depth and thermodynamic properties of an ML, and the cloud core fraction of cumulus (van Stratum et al. 2014). However, the ML energetics is not modeled. The only diagnosed variable that is associated with the intensity of convection is $w^{*}$. Although $w^{*}$ has been proven to be a good proxy of updraft intensity in equilibrium MLs (van Stratum et al. 2014), some doubt has been cast on its validity over nonstationary surface fluxes (van Driel and Jonker 2011). As such, to what extent $w^{*}$ is representative of the updraft strength in different stages of a diurnal cycle is a key question to answer. This motivates us to use the LES to test the validity of $w^{*}$. The LES results will lead us to propose a new convective velocity scale that can successfully approximate the updraft strength in different stages of a diurnal cycle, in particular in the decaying stage (Sorbjan 1997).

\section{a. LES data}

The LES simulation data used in this study are from Sakradzija and Hohenegger (2017). They use the University of California, Los Angeles (UCLA)-LES to perform simulations. The UCLA-LES model comprises the Navier-Stokes equations, the thermodynamic and moisture transport equations, the anelastic continuity equation and the ideal gas law as formulated by Ogura and Phillips (1962). The equations are solved using finite-differences and are discretized over a doubly periodic uniform Arakawa $\mathrm{C}$ grid. The prognostic variables include the wind components, the liquid-water potential temperature (Deardorff 1976), and the totalwater mixing ratio. The transport equations for a number of scalars that describe the microphysical processes are also available, but are not used in this study. The subgrid turbulent fluxes are parameterized using Smagorinsky's (1963) model, while the physical processes such as the surface fluxes and radiation are prescribed. Time stepping is based on a Runge-Kutta third-order iterative method. A directional-split monotone up winding scheme is used for scalar advection, while momentum advection is solved by a directionally split fourth-order centered differences. A more detailed description of the UCLA-LES model is provided in Stevens (2010).

We study a classical continental shallow cumulus case at the SGP site under the aegis of the Department of Energy (DOE) Atmospheric Radiation Measurement (ARM) program on 21 June 1997 (Brown et al. 2002). On this day, shallow cumuli developed on top of an initially clear boundary layer at $\sim 1500$ UTC. Then, forced by surface heat fluxes and entrainment, the subcloud layer deepened and warmed, following a diurnal cycle typical for continental shallow cumulus. The simulation starts at 1130 UTC (0630 local time) and ends at 0200 UTC next day (2100 local time), spanning a whole daytime diurnal cycle. The forcing data are the same with those used in (Brown et al. 2002), which include an early morning sounding from revised radiosonde data and observed surface sensible and latent heat fluxes. The domain size is $51.2 \mathrm{~km} \times 51.2 \mathrm{~km}$. Horizontal and vertical resolutions are $25 \mathrm{~m}$ up to the domain top $(5 \mathrm{~km})$.

In addition to the control case, Sakradzija and Hohenegger (2017) ran four more simulations by varying the surface sensible and latent heat fluxes with total surface fluxes virtually unchanged (Table 1). 
TABLE 1. Information of five LESs with different Bowen ratio.

\begin{tabular}{ccc}
\hline \hline Abbreviation & $\begin{array}{c}\text { Maximum Bowen } \\
\text { ratio }\end{array}$ & $\begin{array}{c}\text { Total surface fluxes } \\
\left(\mathrm{W} \mathrm{m}^{-2}\right)\end{array}$ \\
\hline A-base & 0.36 & 343 \\
A- 0.5 & 0.5 & 340 \\
A-0.1 & 0.11 & 347 \\
A- 0.06 & 0.06 & 348 \\
A- 0.03 & 0.03 & 349 \\
\hline
\end{tabular}

Figure 4 shows the diurnal cycles of the surface forcing parameters (Figs. 4a-c) and the modeled $h$ (Fig. 4d) and the vertical velocity variance integrated from the surface to $h$ (Fig. $4 \mathrm{e}$ ); $h$ is determined as the altitude with minimum buoyancy fluxes in the vertical. Consistent with the idea from Williams and Stanfill (2002) and other earlier studies (Golaz et al. 2001; Stevens 2007), a higher BR favors stronger convection. The enhanced convection deepens the mixed layer via strengthening the entrainment rate. After cloud initiations, $h$ syncs with $z_{b}$. The ML continues to deepen through entrainment, but at a slower pace than the earlier clear stage because of the cumulus mass fluxes that buffer the entrainmentinduced deepening (Medeiros et al. 2005; Neggers et al. 2006). In the decaying stage (after $\sim 1800$ LST), the ML convection calms down although the cloud bases remain high.

\section{b. Examining the validity of $w^{*}$ with LES}

Our objective here is to examine to what degree $w^{*}$ can explain the variability of updrafts in an ML. We use the LES-diagnosed $\sqrt{\left\langle\overline{\left\langle w^{\prime} w^{\prime}\right\rangle}\right.}$, in which $\left\langle\overline{\left.w^{\prime} w^{\prime}\right\rangle}\right.$ is the bulk average of vertical velocity variance over the ML, to represent the "truth" updraft intensity. Empiricism suggests that $w^{*}$ is always larger than $\sqrt{\overline{\left\langle w^{\prime} w\right\rangle}}$ (Lenschow et al. 1980; Stull 2012), which makes physical sense because $w^{*}$ is derived under the assumption of free convection. To make $\sqrt{\overline{\left\langle w^{\prime} w^{\prime}\right\rangle}}$ and $w^{*}$ comparable in terms of absolute value, we multiply $\sqrt{\left\langle w^{\prime} w^{\prime}\right\rangle}$ by an empirical parameter $C=1.78$. The value of $C$ is derived from Lenschow et al.'s (1980) classical formula of the profile of vertical velocity variance that is empirically determined from aircraft measurements (see appendix $\mathrm{C}$ for detail derivation). Earlier studies have confirmed the good performance of this formula in both clear and cloudy conditions (Berg et al. 2017; Brown et al. 2002; Hogan et al. 2009; Lareau et al. 2018; Zheng et al. 2015), and a recent work offers theoretical support for the universality of this formula (Zhou et al. 2019).

We compare $C \sqrt{\left\langle w^{\prime} w^{\prime}\right\rangle}$ with $w^{*}$ for the five runs. As shown in Fig. 5a, the overall agreement is good, but a marked scatter is noticeable. There is a distinctive diurnal dependence. For a given $w^{*}$, the real convection is
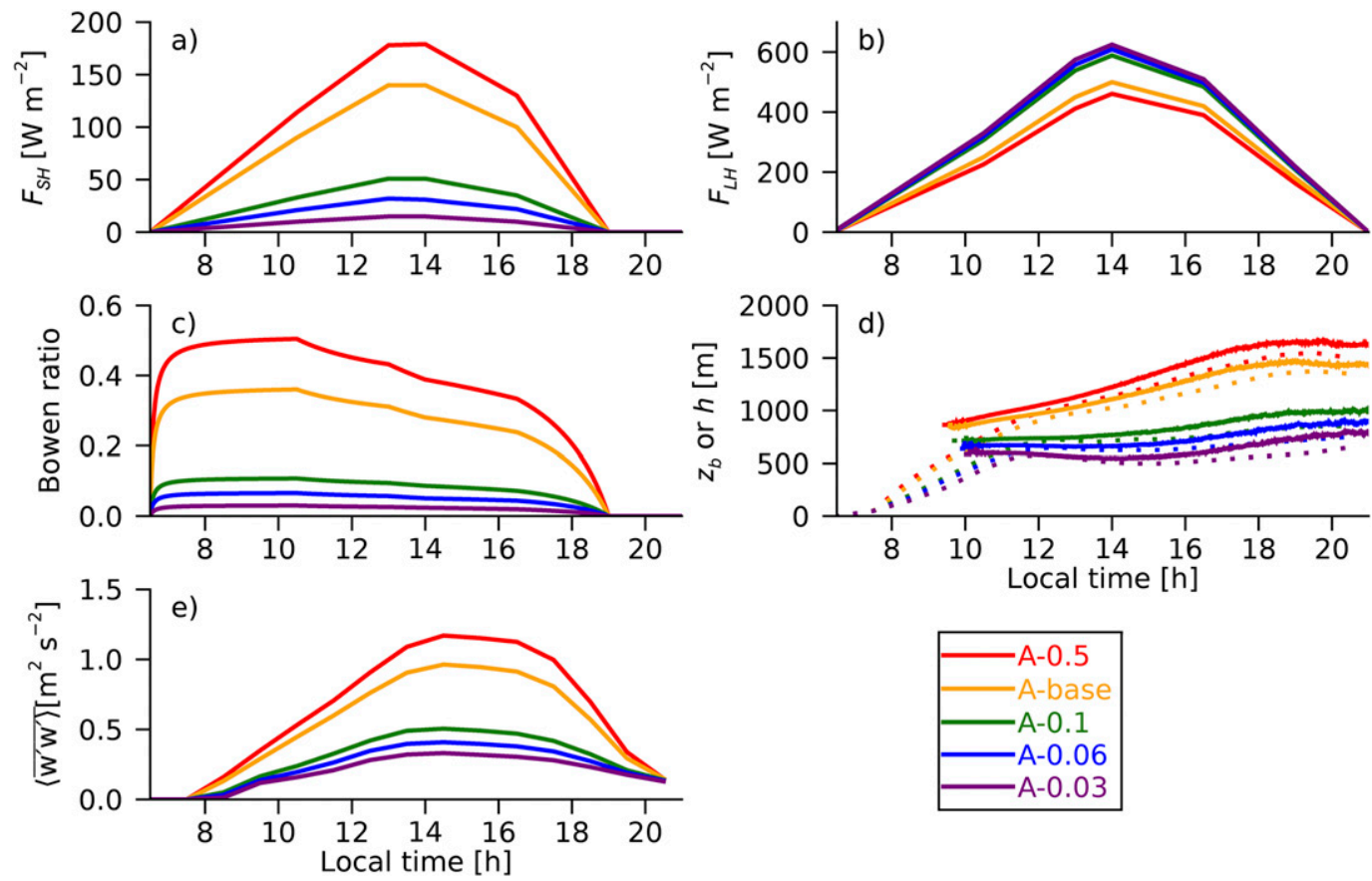

FIG. 4. Time series of the simulated (a) surface sensible heat fluxes, (b) surface latent heat fluxes, (c) Bowen ratio, (d) cloud-base height (solid) and mixed-layer depth (dashed), and (e) vertically averaged vertical velocity variance for the LES ARM case. 

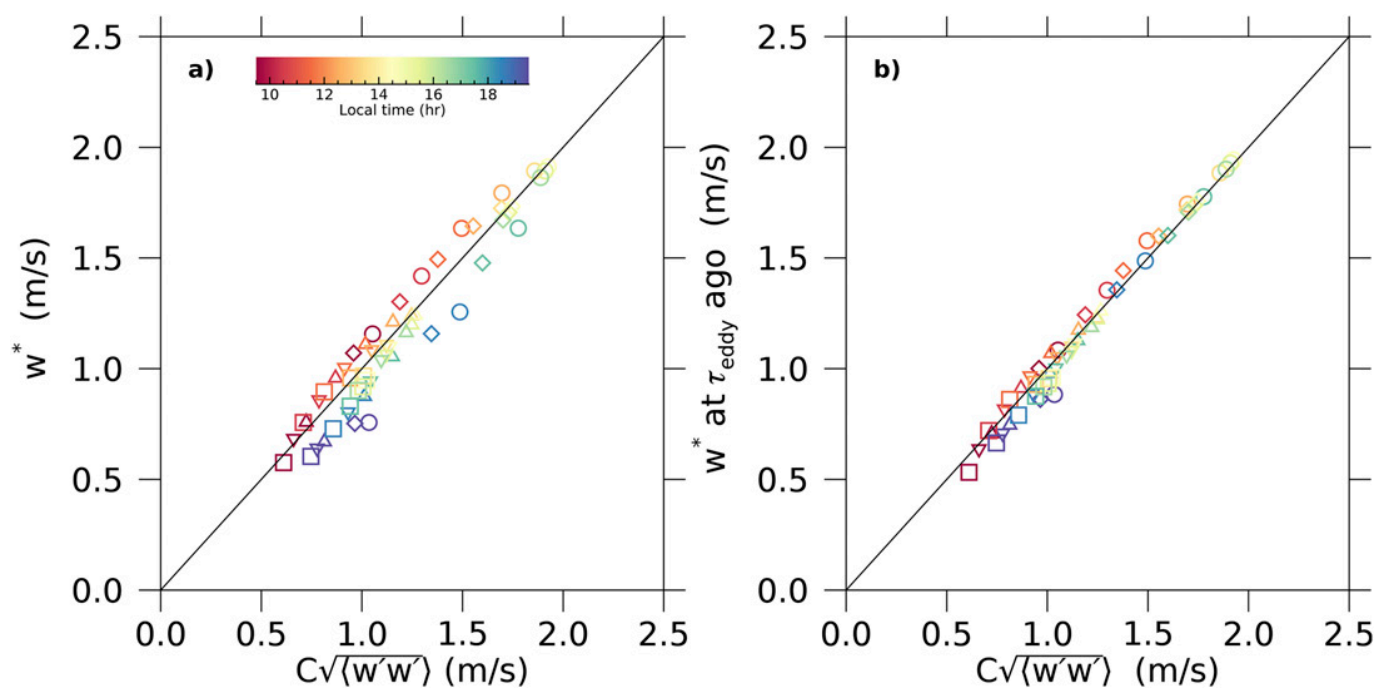

FIG. 5. Comparisons between the LES-simulated $C \sqrt{\overline{\left\langle w^{\prime} w^{\prime}\right\rangle}}$ (abscissa) and (a) instantaneous $w^{*}$ and (b) $w^{*}$ at $0.5 \mathrm{~h}$ ago.

stronger in the evening than in the morning. What causes such a diurnal dependence? As mentioned earlier, the vertical velocity responds to the surface flux forcing at a time scale of several tens of minutes $\left(\tau_{\text {eddy }}\right)$. This means that the vertical velocity does not necessarily dictate the instantaneous surface fluxes, but retains the memory of surface buoyancy flux at $\tau_{\text {eddy }}$ earlier. To examine this "time lag" effect, we use the $F_{B}$ value a half hour earlier, instead of the instantaneous value, to calculate the $w^{*}$. As shown in Fig. 5b, $C \sqrt{\overline{\left\langle w^{\prime} w\right\rangle^{\prime}}}$ agrees better with earlier $w^{*}$ than the instantaneous $w^{*}$ although a weak dependence on local times is still noted.

The above analysis suggests that accounting for the time-lag effect improves the capability of $w^{*}$ in representing the updraft strength for continental shallow convection. Here we propose a new effective convective velocity scale $w_{\mathrm{eff}}^{*}$ to replace $w^{*}$ in the MLM:

$$
w_{\mathrm{eff}}^{*}(t)=\left[\frac{g h}{\theta_{v}} F_{B}\left(t-C \frac{h}{w_{\mathrm{eff}}^{*}}\right)\right]^{1 / 3},
$$

where we quantify the time lag as $h /\left(w_{\mathrm{eff}}^{*} / C\right)=h / \sqrt{\left\langle\overline{\left\langle w^{\prime} w^{\prime}\right\rangle}\right.}$. The terms $h$ and $\sqrt{\overline{\left\langle w^{\prime} w\right\rangle^{\prime}}}$ are considered as the relevant scales for length and vertical velocity, respectively, so that $h / \sqrt{w^{\prime} w^{\prime}}$ represents the time with which surface air parcels circulate through the ML. Because it is an implicit expression, $w_{\text {eff }}^{*}$ needs to be solved by iteration in each time step of an MLM simulation.

\section{c. Validation against LES}

To evaluate the performance of $w_{\mathrm{eff}}^{*}$, we run the MLM for the same ARM SGP case. The model is initialized $1 \mathrm{~h}$ after the beginning time of LES. The LES outputs are extracted to construct the initial thermodynamic state and $h$ for the MLM (see appendix B for detail). A comprehensive validation of the MLM has been conducted in van Stratum et al. (2014) with satisfying results. Here we focus our attention on $h$ and $w_{\text {eff }}^{*}$. Figure 6 shows that the two parameters modeled by the MLM agree reasonably well with their LES equivalents although there is a systematic overestimation of $h$ by $\sim 200 \mathrm{~m}$ for low-BR runs (A-0.1, A-0.06, A-0.03), whose cause will be discussed later. The good performance of $w_{\mathrm{eff}}^{*}$ at evening decaying stage is particularly encouraging. The common issue of conventional $w^{*}$ (dashed lines) in underestimating the decaying-stage convection is well circumvented by using $w_{\text {eff }}^{*}$ instead (Sorbjan 1997).

For each time step, we quantify $\lambda$ as the slope of the best-fit line of updraft speed versus $h$ for the five simulations. The value of $\lambda$ from the MLM agrees well with that from the LES (Fig. 6c). There is a systematic overestimation of $\lambda$ after 1200 LST. This is due to the overestimated $h$ for low-BR cases by the MLM, which steepens the slope of the $w_{\text {eff }}^{*}-h$ relationship.

Despite the overall good performance, a noticeable problem is that the MLM systematically overestimates $h$ for low-BR cases (A-0.1, A-0.06, A-0.03). Such an overestimation occurs after the cloud initiates when the $h$ evolution is controlled by the competition between the entrainment rate and cloud-based mass fluxes $M$, with the former increasing $h$ and the later shoaling it. To find out the reason for the MLM overestimation, we examine $M$ in two models (Fig. 7). We do not examine the entrainment rate because it is not directly retrievable from the LES data. The $M$ is defined as $M=a^{c} w^{c}$, in which 

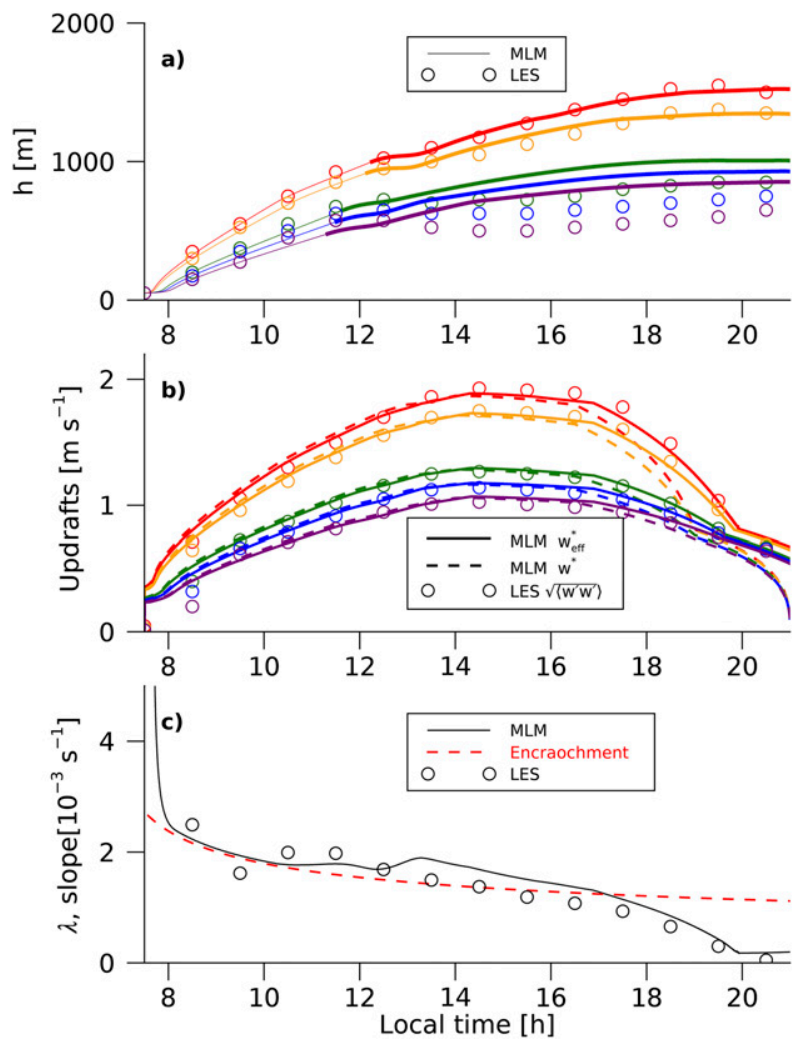

FIG. 6. (a) Comparison of simulated $h$ between MLM (solid line) and LES (open circles). (b) Comparison between the MLMsimulated $w^{*}$ (dashed line), $w_{\text {eff }}^{*}$ (solid line), and LES-simulated $C \sqrt{\overline{\left\langle w^{\prime} w\right\rangle^{\prime}}}$ (open circles). (c) Comparison of the updrafts- $z_{b}$ slopes $(\lambda)$ between MLM (line) and LES (open circles). In (a), the thicker lines represent the period with cumulus. In (c), the red dashed curve is calculated from the "encroachment" theory.

$a^{c}$ and $w^{c}$ are the cloud fraction and vertical velocity at bases of active (or cloud core) clouds. In MLM, both variables are parameterized by boundary layer quantities (see Part I for detail). In LES, we select active buoyant cloud pixels with the vertically integrated $\theta_{v}$ greater than the domain-averaged value by $0.5 \mathrm{~K}$. Cloud-base vertical velocities of these active buoyant pixels are used to compute $M$. In the Fig. 7, both models show an earlier development of $M$ under lower BR, but the initiations of $M$ in MLM are ahead of the LES by about a half hour. Such a shift to earlier times causes the $M$ to be overestimated in the beginning and underestimated late in the afternoon. Overall, the MLM performs reasonably well in reproducing the absolute values and temporal evolutions of $M$. The diurnal means of $M$ from MLM is $0.2-0.4 \mathrm{~cm} \mathrm{~s}^{-1}$ greater than that from LES, suggesting that if only $M$ is considered in $h$ evolution, the MLM shall underestimate $h$. However, as shown by Fig. 6a, the MLM markedly overestimates $h$ for low-BR runs and estimates $h$ well for other runs. This suggests that the

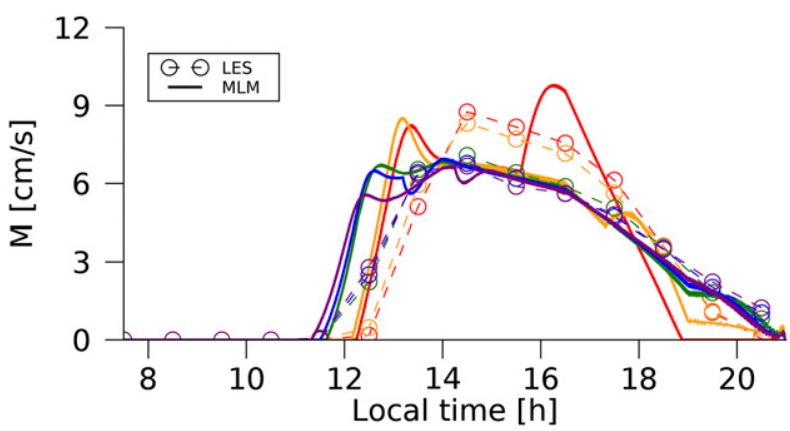

FIG. 7. Temporal evolution of cloud-base mass fluxes simulated by LES (open circles) and by MLM (lines).

MLM must overestimate the entrainment rate so that the excess deepening compensates (or overcompensate in low-BR cases) for the $h$. The reason for the BR-dependence is not straightforward to answer. A good starting point is to examine the buoyancy jump across the ML top, a key parameter that governs the entrainment. This quantity is influenced by the cumulus activities (e.g., lateral mixing of cumulus with the ambient air), which are regulated by $F_{\mathrm{LH}}$. However, an undisputed retrieval of the buoyancy jump from LES data proves to be extremely difficult (Lilly 2002). We leave this as an open question for future research.

\section{Results}

Having confirmed the good performance of the MLM, we use it as the main tool to examine the three hypotheses proposed in the section 2 . The results from the simple encroachment theory suggest that $\lambda$ decreases with time and stability of the background fluid, or $N$, is a key controlling factor of $\lambda$. But the encroachment theory does not account for the penetrative entrainment and, if cumulus emerges, the cloud-base mass fluxes. Both processes impact the budgets of mass, moisture, and energy of an ML. So their impacts on $\lambda$ should be examined.

\section{a. Case study}

Table 2 describes the four MLM simulations conducted for the same ARM SGP case as above. The "high $N$," "dry," and "low $A$ " runs are intended to examine the impacts of atmospheric stability, cloud-base mass fluxes, and efficiency of penetrative entrainment on $\lambda$, respectively.

Figure 8a shows $\lambda$ varying with time for the four sets of simulations. All simulations show $\lambda$ decreasing with time. In addition, $\lambda$ is sensitive to $N$. The value of $\lambda$ is greater when the background fluid is more stable, which is consistent with the result from the encroachment 
TABLE 2. Descriptions of the MLM simulations for the ARM case. In the base case instead of using the piecewise structure of free-atmosphere lapse rates, we use their mean values for simplicity.

\begin{tabular}{cc}
\hline \hline Abbreviation & Description \\
\hline Base & ARM base case: $\Gamma_{\theta}=4.5 \mathrm{~K} \mathrm{~km}^{-1}, \Gamma_{q}=$ \\
& $4.5 \mathrm{~g} \mathrm{~kg}^{-1} \mathrm{~km}^{-1}$ \\
High- $N$ & As in the base case, but $\Gamma_{\theta}=6.5 \mathrm{~K} \mathrm{~km}^{-1}$, \\
& suggesting a more stable background \\
& atmosphere (high $N)$ \\
Dry & Convective mass fluxes $M$ forced to zero \\
& throughout the simulation \\
Low- $A$ & Entrainment efficiency $A=0.15$ reduced \\
& to $A=0.05$ \\
\hline
\end{tabular}

theory. To explore what contributes to the greater $\lambda$, we examine $w_{\text {eff }}^{*}$ versus $h$ at $t=1200$ local time for the four sets of simulations. The difference in $h$ between $\mathrm{BR}=$ 0.5 and $\mathrm{BR}=0.03$, denoted $\Delta h$, is considerably smaller in the "high $N$ " runs than the "base" runs (by 20\%), whereas $\Delta w_{\text {eff }}^{*}$ is only marginally smaller in the "high $N$ " runs (by 7\%). This causes a greater $\lambda$ in the "high $N$ " runs. The same mechanism can be applied for explaining the greater $\lambda$ in "low $A$ " runs than in the "base" runs because reducing the entrainment efficiency slows the growth rate of $h$.

The influence of cumulus mass fluxes is negligible as seen from the near complete overlap between the "base" and "dry" runs (Figs. 8a,b). In principle, the cumulus mass flux can shoal the ML, counteracting a certain extent of the entrainment-induced $h$ deepening, but this effect is much less noticeable than the impacts of atmospheric static stability and entrainment efficiency.

In summary, a case study of MLM shows results consistent with the encroachment theory: $\lambda$ decreases with time and increases with atmospheric stability. The entrainment efficiency $A$ also influences the $\lambda$, but $A$ is an empirical constant and there is no practical meaning of studying it. Prior studies use $A$ values of 0.15 or 0.2 to address a range of questions with satisfactory results (e.g., Stevens 2006). Thus, we just fix it as 0.15 , by following van Stratum et al. (2014).

\section{b. An ensemble of MLM simulations}

To generalize the findings from the case study, we conduct an ensemble of MLM simulations under different combinations of boundary and initial forcing parameters, which are summarized in Table 3. There are a total of 1215 combinations of settings. In each setting, we vary the BR from 0.01 to $9(0.01,0.05,0.1,0.2,0.5,1$, $2,5,9)$ to obtain $\lambda$ and its diurnal cycle. Among these $\sim 10000$ runs, a quarter corresponds to unphysical settings in which $q_{\mathrm{ML}}^{0}$ is greater than the saturation water
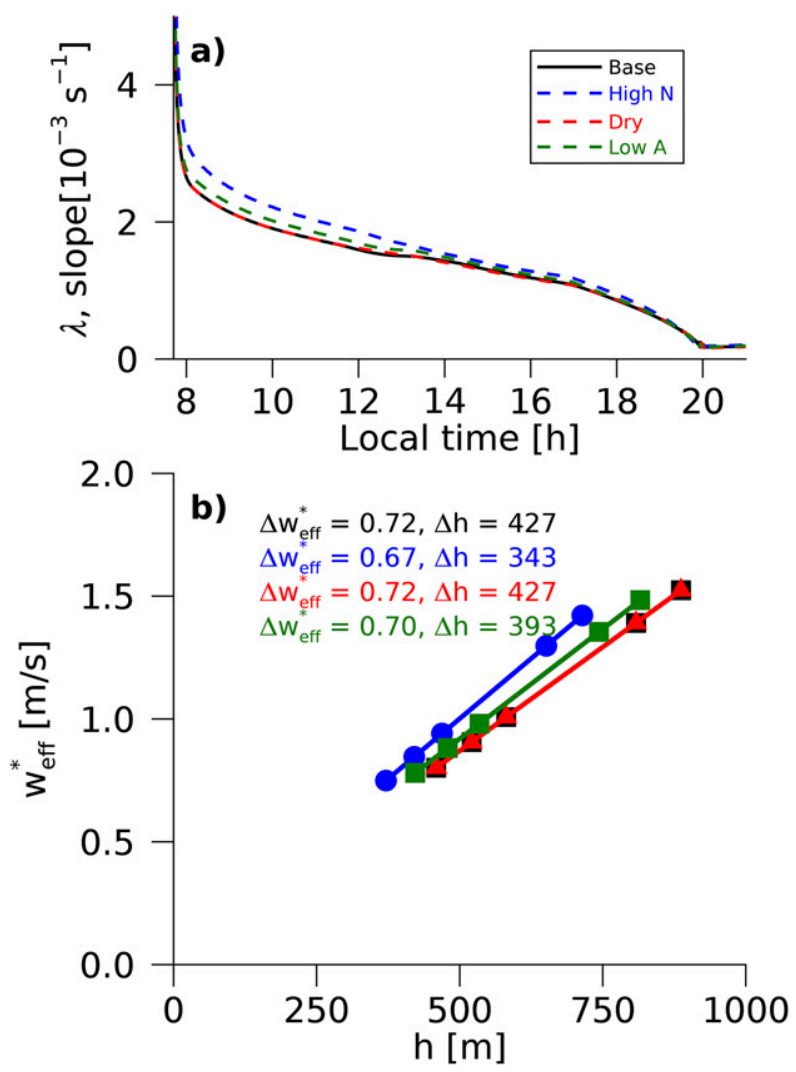

FIG. 8. (a) Diurnal evolution of $\lambda$ and (b) $w_{\text {eff }}^{*}$ vs $h$ at $t=12 \mathrm{~h}$ local time for the four sets of simulations. The difference between $\mathrm{BR}=0.5$ and $\mathrm{BR}=0.03$ runs is denoted with $\Delta$.

vapor mixing ratio for $\theta_{\mathrm{ML}}^{0}$. These cases were removed for analysis. Another quarter of the simulations are clear boundary layers with no occurrence of cumulus at any stage of a diurnal cycle. The remaining $\sim 50 \%$ of the simulations exhibit cumulus-like features: 1) $a^{c}$ remains several percent throughout the day, which is consistent with modeling studies (Brown et al. 2002; Neggers et al. 2004), and 2) $a^{c}$ shows an increase-then-decrease diurnal curve, consistent with empirical observations (Lareau et al. 2018) and LES. This subset of simulations ( $~ 5000$ simulations) were pulled out for further analysis.

Figure 9a shows the diurnal cycle of $\lambda$ for the ensemble simulations. Each line is color-coded by the daily mean $N$. The high- $N$ curves lie systematically above the low- $N$ curves, presenting a rainbow-like pattern, which suggests a dominant role of $N$ in governing $\lambda$. Figure $9 b$ shows $\lambda$ averaged from $t=2$ to $t=14.5 \mathrm{~h}$ as a function of $N$. The $N$ explains more than $99 \%$ variation of the daily mean $\lambda$. Among the six forcing parameters in Table $3, \Gamma_{\theta}$ dominantly contributes to the variability of $\lambda$, as seen from the points segregated by the colors (Fig. 9b). For a given $\Gamma_{\theta}, \Gamma_{q}$ is the major regulator of $\lambda$, as seen from the segregated symbols with the same color in Fig. $9 \mathrm{~b}$. 
TABLE 3. Statistics of the ranges of the perturbed environmental parameters.

\begin{tabular}{lcc}
\hline \hline \multicolumn{1}{c}{ Parameter } & Units & Values \\
\hline$\Gamma_{\theta}$ & $10^{-3} \mathrm{~K} \mathrm{~km}^{-1}$ & $3,4,5,6,7$ \\
$\Gamma_{q}$ & $\mathrm{~g} \mathrm{~kg}^{-1} \mathrm{~km}^{-1}$ & $-3,-5,-7$ \\
Daily maximum surface & $\mathrm{W} \mathrm{m}$ & $500,600,700$ \\
$\quad$ radiative forcing & & \\
Initial $\theta_{\mathrm{ML}}\left(\theta_{\mathrm{ML}}^{0}\right)$ & $\mathrm{K}$ & $290,298,306$ \\
Initial $q_{\mathrm{ML}}\left(q_{\mathrm{ML}}^{0}\right)$ & $\mathrm{g} \mathrm{kg}^{-1}$ & $8,13,18$ \\
Large-scale divergence & $10^{-6} \mathrm{~s}^{-1}$ & $1,5,9$ \\
\hline
\end{tabular}

The results make physical sense because $N$ is primarily determined by $\Gamma_{\theta_{v}}$, a combination of $\Gamma_{\theta}$ and $\Gamma_{q}$. Other forcing parameters have minor impacts on $\lambda$.

It is worthwhile to note that the continental $\lambda$ is systematically larger than the oceanic value of $0.57 \times$ $10^{-3} \mathrm{~s}^{-1}$ (dashed line in Fig. 9a), calculated by that same model in Part I. This is qualitatively consistent with the experimental data (Zheng and Rosenfeld 2015 ) that show a $\sim 20 \%$ higher value of $\lambda$ over land than over the ocean. In Zheng and Rosenfeld (2015), most continental data samples were collected in the early afternoon: $t=6-10 \mathrm{~h}$ (gray shading in Fig. 9a). Within this period, the MLM-simulated $\lambda$ doubles or even triples the oceanic $\lambda$, much greater than the observational land-ocean difference of $\sim 20 \%$. This quantitative inconsistency between the MLM and observations can be contributed by many sources of errors including the limitations of the MLM itself and the myriad errors associated with measurements of updraft by Doppler lidar or radar. For example, much of the observational errors of vertical velocity over the open ocean are caused by ship movements whose influences are impossible to fully correct (V. Ghate 2018, personal communication). As such, it is not easy to deduce how much of this MLM-observation difference is related to each error source. But the conclusion of larger $\lambda$ over land than ocean appears to be robust, given the qualitative consistency between the MLM and observations.

\section{c. Comparison with observations}

We use ground-based observations over the SGP site to examine the theoretical findings for continental shallow cumulus. As introduced in section 2, the observational data are obtained from Zheng and Rosenfeld (2015). There is a total of 209 shallow cumulus cases and each case represents a 3-h segment of measurements. Cloud-base heights and vertical velocities are measured by ceilometer and a vertically pointing Doppler lidar, respectively. For each case, Doppler lidar measurements offer a vertical profile of 3-h volume-weighted mean updrafts $w_{\mathrm{vol}}$. We select the maximum $w_{\mathrm{vol}}$ in the vertical $w_{\mathrm{vol}}^{\max }$ to represents the strength of updrafts for an ML. A practical reason for using the vertical maximum instead of the column mean is that the lidar signal is attenuated considerably near the ML top in particular for deep MLs, rendering the Doppler velocity measurements noisy in the upper portion of an ML. To ensure an "apples to apples" comparison between the
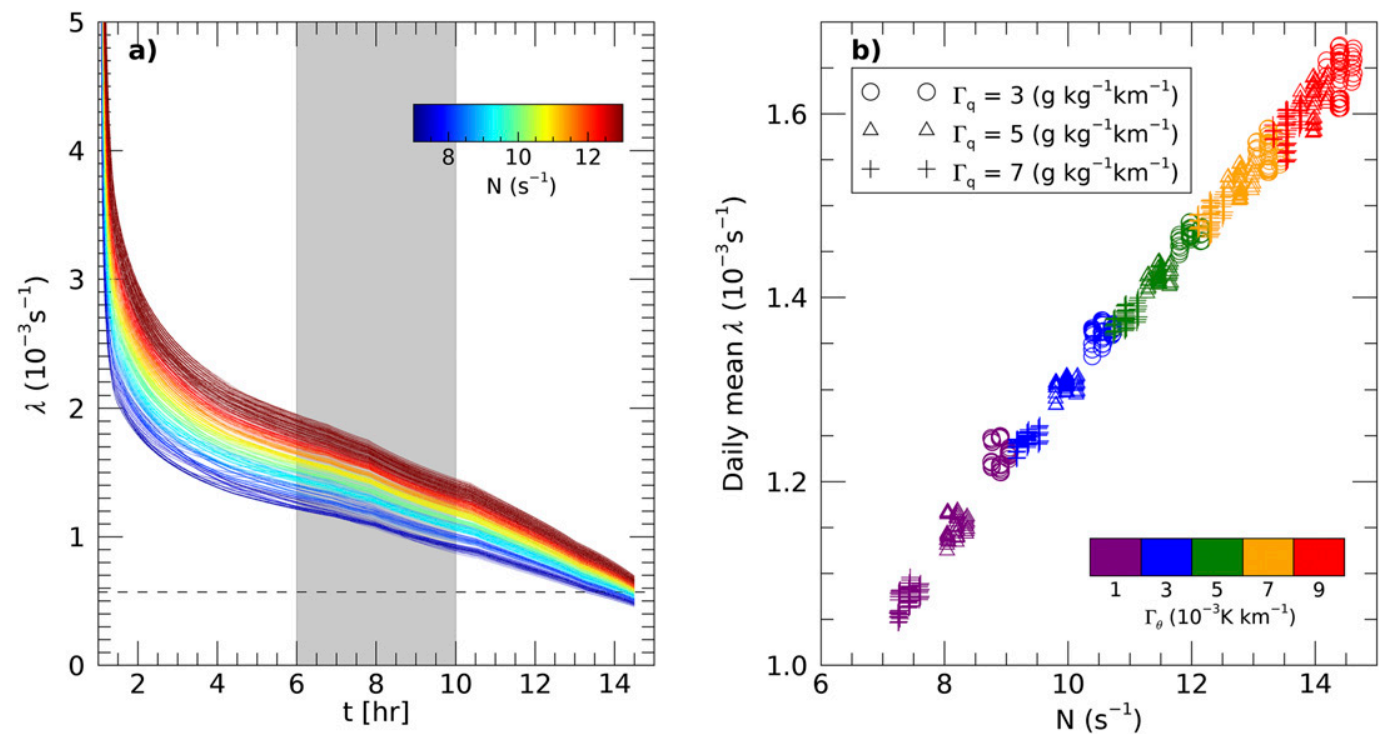

FIG. 9. (a) Temporal evolutions of $\lambda$ for all the MLM simulations. Each line is color coded by $N$. Here $t$ refers to the time after sunrise. (b) Daily mean $\lambda$ vs $N$. The dashed horizontal line marks the oceanic value of $\lambda$ from Part I. The gray shading marks the range of $t$, within which most SGP samples fall (Zheng and Rosenfeld 2015). 
observed $w_{\mathrm{vol}}^{\max }$ and the theoretical $w^{*}$, we divide $w_{\mathrm{vol}}^{\max }$ by 0.67 . The theoretical derivation of the value 0.67 is shown in appendix $\mathrm{D}$. The cases are grouped equally by $t$ and $N$, leading to six equal-sized groups (each containing $\sim 35$ cases). In each group, we linearly fit $w_{\mathrm{vol}}^{\max } / 0.67$ and $z_{b}$. The best-fit lines are forced through the origin. The slope of the best-fitted line is identified as the observationally inferred $\lambda$. Figure 10 shows that the observations (filled dots) agree reasonably well with the theoretical curves: $\lambda$ decreases with time and increases with $N$. Although the observation shows less sensitivity to both factors, the overall match between the theory and observation is encouraging. With controlled $t$ and $N$, the root-mean-square error of the six theoretical $\lambda$ is $0.08 \times 10^{-3} \mathrm{~s}^{-1}$, which amounts to only $\sim 5 \%$ error. Such a small theory-observation difference is somewhat surprising to us given the multiple ad hoc assumptions we make in the theoretical derivations and myriad errors associated with the observational data. We do not rule out the possibility of error cancelations that may obscure potential disagreements. But the overall patterns of the $\lambda$ as functions of $t$ and $N$ are justified by both the theory and observational data, suggesting their robustness.

\section{A conceptual diagram: The importance of time-scale separation}

We have used the encroachment theory, MLM, and observations to answer the three questions posed in the introduction: 1) $\lambda$ decreases with time during daytime, 2) $\lambda$ is greater in more stable atmospheric conditions, and 3) $\lambda$ is greater over land than over ocean. Here, we lay out a conceptual diagram to explain them physically. The most important idea underlying the conceptual diagram is the dramatically different time scales at which updrafts and $h$ respond to the surface flux forcing. Specifically, the updrafts respond to the surface forcing at an eddy turnover time scale $\tau_{\text {eddy }}$ that is only several tens of minutes. The response of $h$ to the surface forcing occurs at a much lower rate. The typical magnitude for daily mean entrainment rate $E$ is several centimeters per second. This corresponds to a response time scale of tens of hours $\left(\tau_{E}=h / E\right)$.

We use the conceptual diagram in Fig. 11 to elaborate on how the magnitude difference between $\tau_{\text {eddy }}$ and $\tau_{E}$ helps answer the three questions. Let us consider an initially equilibrium cumulus-topped boundary layer in which radiative cooling balances the warming by entrainment and surface heat fluxes, and entrainment deepening balances the shallowing by cloud-base mass fluxes and large-scale subsidence (Fig. 11a). Given a fixed solar surface forcing, a perturbed increase in the

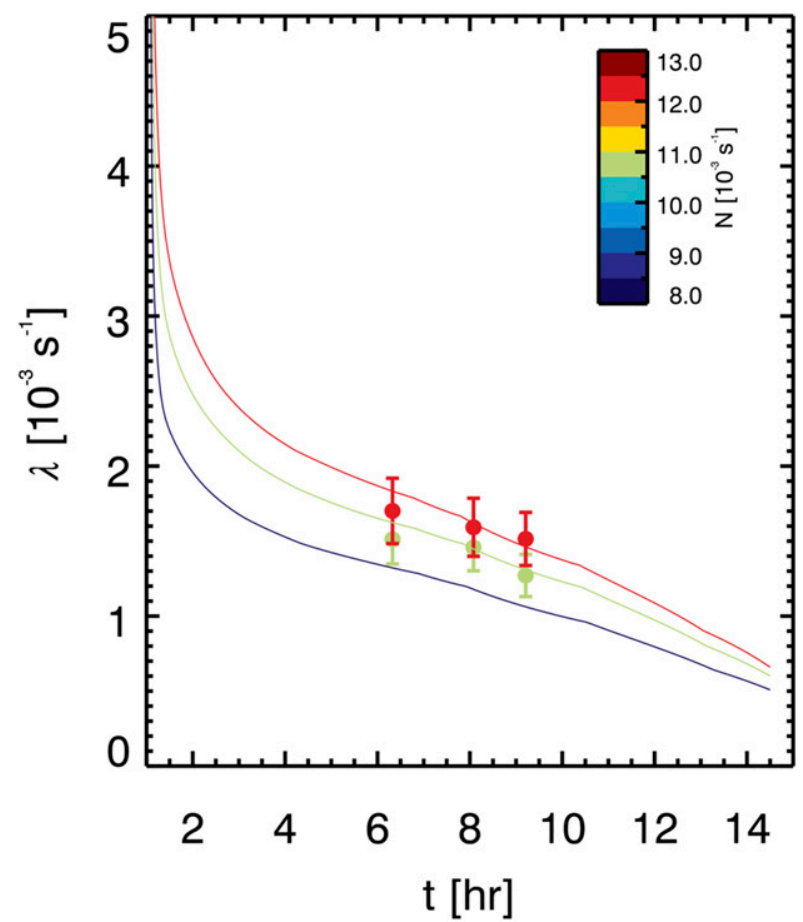

FIG. 10. Comparisons of $\lambda$ between from the MLM (solid lines) and SGP observations (solid dots with error bars). Here $t$ refers to the time after sunrise. The error bars represent uncertainties computed by least squares method.

BR leads to an increase in surface sensible heat flux. As a response, the updraft speed increases immediately because of the short time scale for eddy turnover. In other words, the updraft speed remains approximately slaved to the surface forcing. The strengthened updrafts enhance the entrainment, deepening $h$. Because of $\tau_{E} \gg \tau_{\text {eddy }}, h$ increases at a much slower rate than the updrafts. After several hours, the updrafts have already increased to a level commensurate with the underlying surface fluxes whereas $h$ has only increased by a small degree (Fig. 11b). From the perspective of energy balance, this system is in a transient state because the radiative fluxes divergence across the ML $(h Q)$ is not adequately large to compensate for the excess warming by the increased surface sensible heat flux and by the enhanced entrainment. The energy surplus drives the ML to continue deepening until the radiative flux divergence balances the warming. In the new equilibrium state, $h$ is markedly higher than that in the transient stage whereas the updraft speeds between the two stages are similar. If we use $\Delta w^{*}$ and $\Delta h$ to represent the increases in $w^{*}$ and $h$ with respect to the unperturbed state (Fig. 11a), respectively, $\lambda=\Delta w^{*} / \Delta h$ decreases over time until the new equilibrium. This answers the first question. For the second question, a greater static stability inhibits the ML growth, leading 


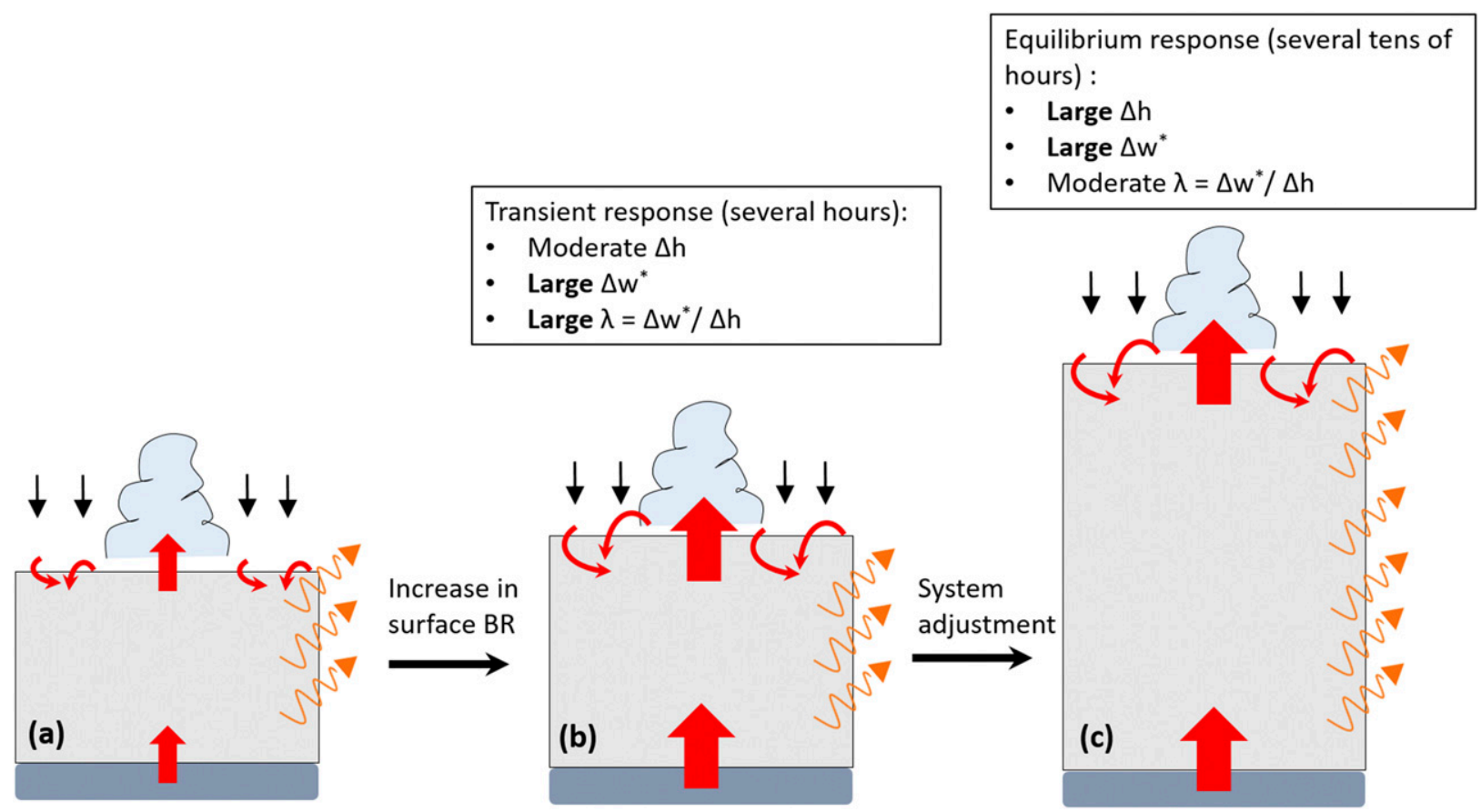

FIG. 11. Cartoon illustrating the different responses of ML depth and updrafts to surface forcing between transient and equilibrium stages.

to a smaller $\Delta h$ and, as a result, a greater $\lambda$ (as shown in Fig. 8b).

The answer to the third question (higher $\lambda$ over land) can also be explained by the same diagram. If the transient (Fig. 11b) and the new equilibrium (Fig. 11c) systems are considered as analogies of continental and maritime boundary layers, respectively, the conclusion that $\lambda$ is greater over land than over ocean is naturally reached. One may argue that over the ocean the BR is too small to drive any marked change in updraft speed. This argument, however, does not apply to marine ML. In a quasi-equilibrium marine ML, what drives changes in $h$ and updraft speed is no longer important; $\lambda$ is only constrained by the conservation of ML energetics (the key message from Part I of this study). In the ML radiative-convective equilibrium, a change in $h$ (e.g., by a change in large-scale subsidence or sea surface temperature) must be associated with a commensurate change in surface buoyancy fluxes and updraft speed, in order to maintain a balance between radiative cooling and warming by surface fluxes and entrainment. The impact of time-scale separation on increasing $\lambda$ (Fig. 11b), which only operates in transient states, is absent in such an equilibrium system. This argument resonates with Fig. 19 in Part I of this study, in which a small perturbation in oceanic BR (by $\sim 0.02$ ) causes an instant increase in $\lambda$ that eventually restores back to the equilibrium $\lambda$ with a smaller value. This suggests that the land-ocean difference in $\lambda$ should not be explained by their differences in the degree of BR variation, but in the degree of equilibrium.

\section{Discussion}

The overall good agreement between the theory and observations leads us to believe that the simple analytical model captures the essence of the problem at hand. However, some physical processes missed by the MLM warrant some discussion here.

First, cloud impacts on evolution of the buoyancy jump across the ML top are not represented in the MLM. The buoyancy jump is expressed as $\Delta \theta_{v}=\theta_{v}^{+}-\theta_{v}^{\mathrm{ML}}$, in which the superscripts " + " and "ML" represent the level just above $h$ and the mixed-layer average, respectively. Determination of $\Delta \theta_{v}$ in the MLM follows the convention for classical clear MLM: $\theta_{v}^{\mathrm{ML}}$ is determined by ML enthalpy budget and $\theta_{v}^{+}$is determined by finding $\theta_{v}$ at the level of $h$ from a given sounding. However, the atmospheric sounding is modified by cumulus convection (Arakawa and Schubert 1974; Stevens 2007). The modifications manifest in two ways. First, moist cloudy air detrains into the dry environment, moistening and cooling the cloud layer. Second, the cumulus mass fluxes trigger an environmental subsidence, forming a convective overturning. The compensating subsidence shapes the inversion strength of the ML top (Fritsch and Chappell 1980). 
Both of these processes can affect $\Delta \theta_{v}$, subsequently influencing the $h$ growth. The less ideal performance of the MLM in $h$ simulation for low-BR cases may be related to these missed processes although the details are not straightforward to identify. A more sophisticated model that parameterizes these processes [such as that developed by Bretherton and Park (2008)] can help enlighten this problem although the greater complexity of their model may prevent heuristic understanding.

Second, the MLM does not account for the coupling between boundary layer processes and the land surface (Betts et al. 1996; Jacobs and De Bruin 1992; Kim and Entekhabi 1998; Margulis and Entekhabi 2001; Santanello et al. 2011; Van Heerwaarden et al. 2009). Surface fluxes are prescribed in this study. However, dry-air entrainment near an ML top can feed back to the surface fluxes by strengthening the surface evaporation (Betts 2004; Van Heerwaarden et al. 2009). This effect can be explored by adding a simple surface model that couples with the MLM through the Penman-Monteith equation (Yin et al. 2015).

Last, mechanically driven turbulence always contributes to the variance of the vertical velocity in a convective boundary layer. In the ARM case studied here, the buoyancy generation of turbulence dominates over wind shear production (Brown et al. 2002). In strong wind conditions, however, the mechanically driven turbulence should play a much more important role, and the results from this study should be applied more carefully.

\section{Conclusions}

We use an analytic mixed-layer model (Neggers et al 2006) to investigate the relationship between cloud-base height and subcloud updraft velocity for continental shallow cumulus (Zheng and Rosenfeld 2015). Consistent with conventional knowledge, we found that an increase in surface Bowen ratio (BR) deepens the subcloud mixed layer (ML) and strengthens the updrafts, leading to a positive covariation between the two variables. The ratio of their respective changes (i.e., the slope of updrafts vs ML depth) is defined as $\lambda$. We have found answers to the three scientific questions posed in the introduction:

1) The value of $\lambda$ decreases from sunrise through sunset.

2) The value of $\lambda$ is greater when the background flow is more stable.

3) The value of $\lambda$ is greater over land than over ocean.

The physical explanations for the three answers are based on the idea of different time scales with which updrafts and ML depth respond to surface flux forcing.
The ML depth responds to a surface forcing at a much slower time scale (entrainment deepening time scale) than the updrafts (eddy turnover time scale). For a given surface solar forcing, an increase in BR enhances the updrafts rapidly whereas the ML deepens at a much slower rate. This leads to the ratio of their respective changes, which is $\lambda$, decreasing with time until the cessation of ML deepening in a new equilibrium. Such a new equilibrium is only possible over oceans. Thus, the equilibrium $\lambda$ over oceans should always be smaller than the transient $\lambda$ over land. In more stable atmospheric conditions, the ML deepens more slowly, leading to a greater $\lambda$.

This study illustrates the importance of the local time, atmospheric stability, and degree of equilibrium in impacting on $\lambda$. This has implications for satellite inference of updrafts from cloud-base height. In Zheng and Rosenfeld (2015), the vast majority of the continental samples were collected in the early afternoon to match the overpass of polar-orbiting satellites. The empirical relationships derived from these early-afternoon samples should not be applied directly to shallow cumulus in the morning or near sunset when $\lambda$ should be markedly different. In addition, the atmospheric static stability is another influential factor for $\lambda$ of continental shallow cumulus. The atmospheric stability obtained from reanalysis data might be used to better inform the satellite inference of updrafts. Over oceans, a quasi-equilibrium state is typically assumed, but to what degree shallow cumulus fields depart from an equilibrium is important to know. Studies examining the degree of equilibrium remain scant. Investigations that synthesize process-level numerical simulations and Lagrangian tracking of cloud fields with remote sensing tools, such as Dagan et al. (2018), are particularly needed.

Acknowledgments. The study was supported by the Department of Energy (DOE) Atmospheric System Research program (DE-SC0018996). The DOE/ARM data are available from www.arm.gov. The IDL codes used for generating the results are available from the first author upon request.

\section{APPENDIX A}

\section{Derivation of $\left(\partial w^{*} / \partial N\right)(\partial N / \partial h)$}

To make the derivations algebraically clearer, we derive $\left[\left(\partial w^{*} / \partial\left(N^{2}\right)\right]\left[\partial\left(N^{2}\right) / \partial h\right]\right.$, which is equal to $\left(\partial w^{*} / \partial N\right)(\partial N / \partial h)$. We can use Eq. (2) to obtain $\partial w^{*} / \partial\left(N^{2}\right)$ :

$$
\frac{\partial w^{*}}{\partial\left(N^{2}\right)}=\frac{1}{3} h(2 t)^{-1 / 3}\left(N^{2}\right)^{-2 / 3} .
$$


To obtain $\partial\left(N^{2}\right) / \partial h$, we insert Eq. (1) into the $N$ formula so that $\gamma_{\theta_{v}}$ is replaced with a formula that contains $h$ :

$$
N^{2}=(2 t) \frac{g F_{B}}{\theta_{v} h^{2}} .
$$

By taking the derivatives of both sides with respective to $h$, we obtain

$$
\frac{\partial\left(N^{2}\right)}{\partial h}=-2 h^{-3}(2 t) \frac{g F_{B}}{\theta_{v}} .
$$

A multiplication of Eqs. (A1) and (A3) gives

$$
\frac{\partial w^{*}}{\partial\left(N^{2}\right)} \frac{\partial\left(N^{2}\right)}{\partial h}=-\frac{2}{3}\left(\frac{g F_{B}}{\theta_{v} h^{2}}\right)\left(\frac{N^{2}}{2 t}\right)^{-2 / 3},
$$

in which the term $g F_{B} /\left(\theta_{v} h^{2}\right)$ can be replaced by $N^{2} /(2 t)$ through Eq. (A2), leading to

$$
\frac{\partial w^{*}}{\partial\left(N^{2}\right)} \frac{\partial\left(N^{2}\right)}{\partial h}=-\frac{2}{3}\left(\frac{N^{2}}{2 t}\right)^{1 / 3} .
$$

\section{APPENDIX B}

\section{Description of the MLM Simulation}

The model has three prognostic equations for $h, q_{\mathrm{ML}}$, and $\theta_{\mathrm{ML}}$, which describe the budgets of mass, moisture, and enthalpy, respectively. It has to be distinguished from another cumulus analytic model (Albrecht et al. 1979) that includes the cloud layer in $h$. The current model parameterizes the cumulus-core area fraction and cumulus mass fluxes with diagnostics of the subcloud layer. The diagnosed cumulus mass fluxes, in turn, influence the subcloud layer development via the regulating mass budgets (van Stratum et al. 2014). The surface fluxes are parameterized by scalar gradients near surface (bulk transfer concept). The entrainment velocity is quantified as buoyancy fluxes at the ML top (a constant fraction of surface buoyancy fluxes) divided by the vertical buoyancy jump at the ML top. A consequence of the interactions between the cumulus mass fluxes and subcloud layer is that the bases of the cumuli always find itself adjacent to the top of $h$ so that the closeness between $z_{b}$ and $h$ is an emergent behavior of the system instead of being assumed in some analytic models (Albrecht et al. 1979). The detail of the equations for the analytic model could be found in Part I or in (Neggers et al. 2006).

The initial and boundary conditions for simulating the ARM case are the same with that in van Stratum et al. (2014) (see their Table B1). The only difference is the initial $h$, for which our LESs show a lower $h=50 \mathrm{~m}$ than that from their study (initial $h=140 \mathrm{~m}$ ). The radiative cooling, advections of temperature and moisture, and large-scale subsidence are set to zero for simplicity. These parameters are important for marine cumulus clouds at steady state, but they prove to be much less essential for the simulations of continental shallow cumulus at a diurnal time scale over which the surface forcing is dominant over these weakly forcing processes (Brown et al. 2002; Sakradzija and Hohenegger 2017).

\section{APPENDIX C}

\section{Conversion between $\sqrt{\overline{\left\langle w^{\prime 2}\right\rangle}}$ and $\boldsymbol{w}_{*}$}

Lenschow et al. (1980) show that

$$
\frac{\overline{w^{\prime 2}}(z)}{w_{*}^{2}}=1.8\left(\frac{z}{z_{i}}\right)^{2 / 3}\left(1-0.8 \frac{z}{z_{i}}\right)^{2} .
$$

By integrating the right side from $z / z_{i}=0$ to 1 , we obtain

$$
\frac{\overline{\left\langle w^{\prime 2}\right\rangle}}{w_{*}^{2}}=\frac{1}{3.18} .
$$

Taking the square root of both sides yields

$$
1.78 \sqrt{\overline{\left\langle w^{\prime 2}\right\rangle}}=w_{*} .
$$

\section{APPENDIX D}

\section{Conversion from Volume-Weighted $w_{\mathrm{vol}}^{\max }$ to $\boldsymbol{w}_{*}$}

To convert volume-weighted $w_{\mathrm{vol}}^{\max }$ to $w_{*}$, two steps are needed: 1$)$ converting volume-weighted mean vertical velocity $w_{\mathrm{vol}}$ to vertical velocity variance $\overline{w^{2}}$ and 2) converting vertically maximum vertical velocity variance $\overline{w^{12}}$ max to $w_{*}$ (the maximum $w_{\mathrm{vol}}$ and maximum $\overline{w^{\prime 2}}$ should occur at the same altitude). The second step is readily available from Lenschow et al.'s (1980) empirical formula, which gives $\overline{w^{\prime 2}}{ }_{\text {max }} / w_{*}^{2}=0.47$.

The conversion between $w_{\mathrm{vol}}$ and $\overline{w^{2}}$ can be solved analytically by assuming a normal distribution of vertical velocity. The normal-distribution assumption is certainly oversimplified because the real vertical velocity spectrum is positively skewed in a typical convective boundary layer (Ansmann et al. 2010; Berg et al. 2017; Lareau et al. 2018; Moeng and Rotunno 1990). Given that the variance and skewness are two independent 
statistical parameters, ignoring the skewness should not considerably affect the results.

We define $w_{\mathrm{vol}}$ as

$$
w_{\mathrm{vol}}=\frac{\int_{0}^{+\infty} n(w) w^{2} d w}{\int_{0}^{+\infty} n(w) w d w},
$$

in which $n(w)$ is the distribution function of $w$. By assuming a normal distribution of $n(w)=\left(1 / \sqrt{2 \pi \sigma^{2}}\right) e^{-w^{2} / 2 \sigma}$, Eq. (D1) can be solved analytically:

$$
w_{\mathrm{vol}}=\sqrt{\frac{\pi \sigma}{2}} .
$$

In a normally distributed vertical velocity field, we have $\sigma^{2}=\overline{w^{\prime 2}}$, which turns Eq. (D2) into

$$
\frac{w_{\mathrm{vol}}}{\sqrt{\frac{w^{2}}{2}}}=\sqrt{\frac{\pi}{2}} .
$$

\section{REFERENCES}

Albrecht, B. A., A. K. Betts, W. H. Schubert, and S. K. Cox, 1979: Model of the thermodynamic structure of the trade-wind boundary layer: Part I. Theoretical formulation and sensitivity tests. J. Atmos. Sci., 36, 73-89, https://doi.org/10.1175/15200469(1979)036<0073:MOTTSO >2.0.CO;2.

Ansmann, A., J. Fruntke, and R. Engelmann, 2010: Updraft and downdraft characterization with Doppler lidar: Cloud-free versus cumuli-topped mixed layer. Atmos. Chem. Phys., 10, 7845-7858, https://doi.org/10.5194/acp-10-7845-2010.

Arakawa, A., and W. H. Schubert, 1974: Interaction of a cumulus cloud ensemble with the large-scale environment, part I. J. Atmos. Sci., 31, 674-701, https://doi.org/10.1175/15200469(1974)031<0674:IOACCE > 2.0.CO;2.

Berg, L. K., R. K. Newsom, and D. D. Turner, 2017: Year-long vertical velocity statistics derived from Doppler lidar data for the continental convective boundary layer. J. Appl. Meteor. Climatol., 56, 2441-2454, https://doi.org/10.1175/ JAMC-D-16-0359.1.

Betts, A. K., 2000: Idealized model for equilibrium boundary layer over land. J. Hydrometeor., 1, 507-523, https://doi.org/10.1175/ 1525-7541(2000)001<0507:IMFEBL > 2.0.CO;2.

, 2004: Understanding hydrometeorology using global models. Bull. Amer. Meteor. Soc., 85, 1673-1688, https://doi.org/ 10.1175/BAMS-85-11-1673.

_ J. H. Ball, A. C. Beljaars, M. J. Miller, and P. A. Viterbo, 1996: The land surface-atmosphere interaction: A review based on observational and global modeling perspectives. J. Geophys. Res., 101, 7209-7225, https://doi.org/10.1029/ 95JD02135.

Bretherton, C. S., and S. Park, 2008: A new bulk shallow-cumulus model and implications for penetrative entrainment feedback on updraft buoyancy. J. Atmos. Sci., 65, 2174-2193, https:// doi.org/10.1175/2007JAS2242.1.

Brown, A., and Coauthors, 2002: Large-eddy simulation of the diurnal cycle of shallow cumulus convection over land
Quart. J. Roy. Meteor. Soc., 128, 1075-1093, https://doi.org/ 10.1256/003590002320373210.

Dagan, G., I. Koren, O. Altaratz, and Y. Lehahn, 2018: Shallow convective cloud field lifetime as a key factor for evaluating aerosol effects. iScience, 10, 192-202, https://doi.org/10.1016/ J.ISCI.2018.11.032.

Deardorff, J. W., 1970: Convective velocity and temperature scales for the unstable planetary boundary layer and for Rayleigh convection. J. Atmos. Sci., 27, 1211-1213, https://doi.org/ 10.1175/1520-0469(1970)027<1211:CVATSF > 2.0.CO;2.

_ 1976: Usefulness of liquid-water potential temperature in a shallow-cloud model. J. Appl. Meteor., 15, 98-102, https://doi.org/ 10.1175/1520-0450(1976)015<0098:UOLWPT>2.0.CO;2.

Donner, L. J., T. A. O'Brien, D. Rieger, B. Vogel, and W. F. Cooke, 2016: Are atmospheric updrafts a key to unlocking climate forcing and sensitivity? Atmos. Chem. Phys., 16, 12 983-12 992, https://doi.org/10.5194/acp-16-12983-2016.

Emanuel, K. A., 1991: A scheme for representing cumulus convection in large-scale models. J. Atmos. Sci., 48, 2313-2329, https:// doi.org/10.1175/1520-0469(1991)048<2313:ASFRCC >2.0.CO;2. , 1994: Atmospheric Convection. Oxford University Press, $580 \mathrm{pp}$.

Fritsch, J., and C. Chappell, 1980: Numerical prediction of convectively driven mesoscale pressure systems. Part I: Convective parameterization. J. Atmos. Sci., 37, 1722-1733, https://doi.org/ 10.1175/1520-0469(1980)037<1722:NPOCDM >2.0.CO;2.

Gentine, P., D. Entekhabi, A. Chehbouni, G. Boulet, and B. Duchemin, 2007: Analysis of evaporative fraction diurnal behaviour. Agric. For. Meteor., 143, 13-29, https://doi.org/ 10.1016/j.agrformet.2006.11.002.

, - — , and J. Polcher, 2011: The diurnal behavior of evaporative fraction in the soil-vegetation-atmospheric boundary layer continuum. J. Hydrometeor., 12, 1530-1546, https:// doi.org/10.1175/2011JHM1261.1.

, A. A. Holtslag, F. D'Andrea, and M. Ek, 2013: Surface and atmospheric controls on the onset of moist convection over land. J. Hydrometeor., 14, 1443-1462, https://doi.org/10.1175/ JHM-D-12-0137.1.

Golaz, J.-C., H. Jiang, and W. R. Cotton, 2001: A large-eddy simulation study of cumulus clouds over land and sensitivity to soil moisture. Atmos. Res., 59-60, 373-392, https://doi.org/ 10.1016/S0169-8095(01)00113-2.

Hartmann, D. L., M. E. Ockert-Bell, and M. L. Michelsen, 1992: The effect of cloud type on Earth's energy balance: Global analysis. J. Climate, 5, 1281-1304, https://doi.org/10.1175/15200442(1992)005<1281:TEOCTO > 2.0.CO;2.

Hogan, R. J., A. L. Grant, A. J. Illingworth, G. N. Pearson, and E. J. O'Connor, 2009: Vertical velocity variance and skewness in clear and cloud-topped boundary layers as revealed by Doppler lidar. Quart. J. Roy. Meteor. Soc., 135, 635-643, https://doi.org/10.1002/qj.413.

Jacobs, C., and H. De Bruin, 1992: The sensitivity of regional transpiration to land-surface characteristics: Significance of feedback. J. Climate, 5, 683-698, https://doi.org/10.1175/15200442(1992)005<0683:TSORTT>2.0.CO;2.

Jeevanjee, N., P. Hassanzadeh, S. Hill, and A. Sheshadri, 2017: A perspective on climate model hierarchies. J. Adv. Model. Earth Syst., 9, 1760-1771, https://doi.org/10.1002/2017MS001038.

Kim, C., and D. Entekhabi, 1998: Feedbacks in the land-surface and mixed-layer energy budgets. Bound.-Layer Meteor., 88, 1-21, https://doi.org/10.1023/A:1001094008513.

Lareau, N. P., Y. Zhang, and S. A. Klein, 2018: Observed boundary layer controls on shallow cumulus at the ARM Southern 
Great Plains site. J. Atmos. Sci., 75, 2235-2255, https://doi.org/ 10.1175/JAS-D-17-0244.1.

Lenschow, D., J. C. Wyngaard, and W. T. Pennell, 1980: Mean-field and second-moment budgets in a baroclinic, convective boundary layer. J. Atmos. Sci., 37, 1313-1326, https://doi.org/ 10.1175/1520-0469(1980)037<1313:MFASMB >2.0.CO;2.

Lilly, D. K., 2002: Entrainment into mixed layers. Part I: Sharpedged and smoothed tops. J. Atmos. Sci., 59, 3340-3352, https://doi.org/10.1175/1520-0469(2002)059<3340:EIMLPI> 2.0.CO;2.

Liu, S., and X.-Z. Liang, 2010: Observed diurnal cycle climatology of planetary boundary layer height. J. Climate, 23, 5790-5809, https://doi.org/10.1175/2010JCLI3552.1.

Margulis, S. A., and D. Entekhabi, 2001: Feedback between the land surface energy balance and atmospheric boundary layer diagnosed through a model and its adjoint. J. Hydrometeor., 2, 599-620, https://doi.org/10.1175/1525-7541(2001)002<0599: FBTLSE $>2.0 . C O ; 2$.

Medeiros, B., A. Hall, and B. Stevens, 2005: What controls the mean depth of the PBL? J. Climate, 18, 3157-3172, https:// doi.org/10.1175/JCLI3417.1.

Miralles, D. G., A. J. Teuling, C. C. Van Heerwaarden, and J. V.-G. De Arellano, 2014: Mega-heatwave temperatures due to combined soil desiccation and atmospheric heat accumulation. Nat. Geosci., 7, 345-349, https://doi.org/10.1038/ngeo2141.

Moeng, C.-H., and R. Rotunno, 1990: Vertical-velocity skewness in the buoyancy-driven boundary layer. J. Atmos. Sci., 47, 1149-1162, https://doi.org/10.1175/1520-0469(1990)047<1149: VVSITB $>2.0 . \mathrm{CO} ; 2$.

Neggers, R., A. Siebesma, G. Lenderink, and A. Holtslag, 2004: An evaluation of mass flux closures for diurnal cycles of shallow cumulus. Mon. Wea. Rev., 132, 2525-2538, https://doi.org/ 10.1175/MWR2776.1.

— B. B. Stevens, and J. D. Neelin, 2006: A simple equilibrium model for shallow-cumulus-topped mixed layers. Theor. Comput. Fluid Dyn., 20, 305-322, https://doi.org/10.1007/ s00162-006-0030-1.

Ogura, Y., and N. A. Phillips, 1962: Scale analysis of deep and shallow convection in the atmosphere. J. Atmos. Sci., 19, 173-179, https://doi.org/10.1175/1520-0469(1962)019<0173: SAODAS $>2.0 . \mathrm{CO} ; 2$.

Sakradzija, M., and C. Hohenegger, 2017: What determines the distribution of shallow convective mass flux through a cloud base? J. Atmos. Sci., 74, 2615-2632, https://doi.org/10.1175/ JAS-D-16-0326.1.

Santanello, J. A., Jr., M. A. Friedl, and W. P. Kustas, 2005: An empirical investigation of convective planetary boundary layer evolution and its relationship with the land surface. J. Appl. Meteor., 44, 917-932, https://doi.org/10.1175/JAM2240.1.

,$- \ldots$, and M. B. Ek, 2007: Convective planetary boundary layer interactions with the land surface at diurnal time scales: Diagnostics and feedbacks. J. Hydrometeor., 8, 1082-1097, https://doi.org/10.1175/JHM614.1.

— C. D. Peters-Lidard, and S. V. Kumar, 2011: Diagnosing the sensitivity of local land-atmosphere coupling via the soil moisture-boundary layer interaction. J. Hydrometeor., 12, 766-786, https://doi.org/10.1175/JHM-D-10-05014.1.

Smagorinsky, J., 1963: General circulation experiments with the primitive equations: I. The basic experiment. Mon. Wea. Rev.,
91, 99-164, https://doi.org/10.1175/1520-0493(1963)091<0099: GCEWTP $>2.3 . \mathrm{CO} ; 2$.

Sorbjan, Z., 1997: Decay of convective turbulence revisited. Bound.-Layer Meteor., 82, 503-517, https://doi.org/10.1023/ A:1000231524314.

Stevens, B., 2006: Bulk boundary-layer concepts for simplified models of tropical dynamics. Theor. Comput. Fluid Dyn., 20 , 279-304, https://doi.org/10.1007/s00162-006-0032-z.

_ 2007: On the growth of layers of nonprecipitating cumulus convection. J. Atmos. Sci., 64, 2916-2931, https://doi.org/ 10.1175/JAS3983.1.

_ 2010: Introduction to UCLA-LES: Version 3.2.1. MPI Doc., 20 pp., https://www.mpimet.mpg.de/fileadmin/atmosphaere/ herz/les_doc.pdf.

Stull, R. B., 2012: An Introduction to Boundary Layer Meteorology. Vol. 13. Springer Science and Business Media, 670 pp.

Tselioudis, G., W. Rossow, Y. Zhang, and D. Konsta, 2013: Global weather states and their properties from passive and active satellite cloud retrievals. J. Climate, 26, 7734-7746, https:// doi.org/10.1175/JCLI-D-13-00024.1.

van Driel, R., and H. J. Jonker, 2011: Convective boundary layers driven by non-stationary surface heat fluxes. J. Atmos. Sci., 68 , 727-738, https://doi.org/10.1175/2010JAS3643.1.

Van Heerwaarden, C. C., J. Vilà-Guerau de Arellano, A. F. Moene, and A. A. Holtslag, 2009: Interactions between dry-air entrainment, surface evaporation and convective boundarylayer development. Quart. J. Roy. Meteor. Soc. 135, 12771291, https://doi.org/10.1002/QJ.431.

van Stratum, B. J., J. Vilá-Guerau de Arellano, C. C. van Heerwaarden, and H. G. Ouwersloot, 2014: Subcloud-layer feedbacks driven by the mass flux of shallow cumulus convection over land. J. Atmos. Sci., 71, 881-895, https://doi.org/ 10.1175/JAS-D-13-0192.1.

Williams, E., and S. Stanfill, 2002: The physical origin of the landocean contrast in lightning activity. C. R. Phys., 3, 1277-1292, https://doi.org/10.1016/S1631-0705(02)01407-X.

Wood, R., and C. S. Bretherton, 2006: On the relationship between stratiform low cloud cover and lower-tropospheric stability. J. Climate, 19, 6425-6432, https://doi.org/10.1175/JCLI3988.1.

Yin, J., J. D. Albertson, J. R. Rigby, and A. Porporato, 2015: Land and atmospheric controls on initiation and intensity of moist convection: CAPE dynamics and LCL crossings. Water Resour. Res., 51, 8476-8493, https://doi.org/10.1002/ 2015 WR017286.

Zheng, Y., 2019: Theoretical understanding of the linear relationship between convective updrafts and cloud-base height for shallow cumulus clouds. Part I: Maritime conditions. J. Atmos. Sci., 76, 2539-2558, https://doi.org/10.1175/JAS-D-18-0323.1.

— , and D. Rosenfeld, 2015: Linear relation between convective cloud base height and updrafts and application to satellite retrievals. Geophys. Res. Lett., 42, 6485-6491, https://doi.org/ 10.1002/2015GL064809.

,$- \ldots$, and Z. Li, 2015: Satellite inference of thermals and cloud-base updraft speeds based on retrieved surface and cloud-base temperatures. J. Atmos. Sci., 72, 2411-2428, https:// doi.org/10.1175/JAS-D-14-0283.1.

Zhou, B., S. Sun, J. Sun, and K. Zhu, 2019: The universality of the normalized vertical velocity variance in contrast to the horizontal velocity variance in the convective boundary layer. J. Atmos. Sci., 76, 1437-1456, https://doi.org/10.1175/JAS-D-18-0325.1. 